BULLETIN Bulletin hispanique

HISPANIQUE Université Michel de Montaigne Bordeaux

$119-2 \mid 2017$

La Égloga renacentista en el Reino de Nápoles

\title{
Sulle egloghe neolatine di Girolamo Borgia
}

Gallicana

\section{Claudia Corfiati}

\section{(2) OpenEdition}

Journals

Edizione digitale

URL: http://journals.openedition.org/bulletinhispanique/5055

DOI: 10.4000/bulletinhispanique.5055

ISSN: 1775-3821

\section{Editore}

Presses universitaires de Bordeaux

\section{Edizione cartacea}

Data di pubblicazione: 1 décembre 2017

Paginazione: 477-494

ISBN: 979-10-300-0218-8

ISSN: 0007-4640

Notizia bibliografica digitale

Claudia Corfiati, « Sulle egloghe neolatine di Girolamo Borgia », Bulletin hispanique [En ligne], 119-2 |

2017, mis en ligne le 28 décembre 2020, consulté le 13 janvier 2021. URL : http://

journals.openedition.org/bulletinhispanique/5055 ; DOI : https://doi.org/10.4000/bulletinhispanique. 5055 


\section{Sulle egloghe neolatine di Girolamo Borgia: Gallicana}

\section{Claudia Corfiati \\ Università di Bari}

En partant de quelques réflexions sur la transmission des vers de Girolamo Borgia, cet article pose les bases d'une étude de ses églogues latines. Il propose en particulier une lecture de la pièce en vers qui ouvre les Solatia aetatis calamitosa, un dyptique bucolique dédié à Vittoria Colonna dans lequel l'auteur prête sa voix au maître Giovanni Pontano.

Mots-clés : poésie pastorale, Giovanni Pontano, Vittoria Colonna.

Partiendo de unas consideraciones sobre la transmisión de los versos de Girolamo Borgia, en el presente ensayo se ponen las bases para un estudio de las églogas latinas del autor. En particular se propone una lectura de la composición que encabeza los Solatia aetatis calamitosae, un díptico bucólico dedicado a Vittoria Colonna, en el que el autor da voz al maestro Giovanni Pontano.

Palabras clave: bucólica, Giovanni Pontano, Vittoria Colonna.

This paper aims to begin a study on the manuscript tradition of Girolamo Borgia's carmina. Focusing in particular on his pastoral poems, we propose an analysis of the first carmen of the Solatia aetatis calamitosae, a bucolic diptych dedicated to Vittoria Colonna, where the author gives voice to his maestro Giovanni Pontano.

Keywords: pastoral poetry, Giovanni Pontano, Vittoria Colonna. 
eggendo le pagine - pur imprecise e spesso fallaci- che Girolamo Borgia
il giovane volle premettere alla raccolta dei Carmina Lyrica et Heroica del suo avo, edita nel $1666^{1}$, dopo più di un secolo di completo oblio, si ha l'impressione di sfogliare un romanzo di formazione scritto a bella posta per rappresentare gli ideali dell'aristocrazia napoletana tra la fine del Quattrocento e gli inizi del secolo successivo: educazione umanistica "pontaniana», esercizio delle armi e della cavalleria (nel Regno prima, in Italia dopo), ruolo di precettore presso nobili famiglie e di cortigiano in curia, dignità ecclesiastica di alto livello, ritiro dalla vita pubblica, che cela un impegno letterario assiduo nonché appassionato $^{2}$. La bibliografia critica, fino ad oggi, si è fermata sull'attività di scrittore di storia del Borgia, riconoscendo alle sue pagine, ancora inedite, un ruolo di assoluta importanza, per la posizione «stravagante» dell'autore nell'ambito della storiografia moderna ${ }^{3}$; mentre nulla è stato ancora detto a proposito della sua poesia ${ }^{4}$. In questa sede pertanto è mia intenzione avviare una ricerca sui versi del Borgia, partendo dalla sua esperienza bucolica.

Girolamo sicuramente si avvicinò alla scrittura poetica fin da giovanissimo, anzi credo che sia lecito immaginarlo esercitarsi in versi fin dagli anni in cui

1. Hieronymi Borgiae Massae Lubrensis Episcopi, Carmina Lyrica et Heroica quae extant, Venetiis, Ex Typographia Iacobi Zattoni, 1666. Sul pronipote, autore di liriche in volgare, si veda Alessia Scognamiglio, "Girolamo Borgia e la sua raccolta lirica manoscritta», Bollettino del Centro di Studi Vichiani, no 33, 2003, pp. 287-300.

2. Sulla biografia di Girolamo Borgia cf. Gianni Ballistrieri, «Borgia, Girolamo», in Dizionario Biografico degli Italiani, Roma, Istituto della Enciclopedia Italiana, 1970, t. XII, pp. 721-724 e Mauro de Nichilo, «Capitoli Borgiani», Annali della Facoltà di Lettere e Filosofia dell'Università degli studi di Bari, 1989, t. 32, pp. 151-209.

3. Cf. Mauro de Nichilo, «Dal Pontano al Giovio: L'Historia di Girolamo Borgia», in La storiografia umanistica. Atti del convegno (Messina, 22-25 ottobre 1987), Messina, Sicania, 1992, t. I/2, pp. 699-730, Id., "Un coetaneo dei Gaurico: Girolamo Borgia», in A. Granese, S. Martelli, E. Spinelli (eds.), I Gaurico e il Rinascimento meridionale. Atti del Convegno di studi, Montecorvino-Rovella, 10-12 aprile 1988, Salerno, Centro Studi sull'Umanesimo Meridionale, 1992, pp. 372-404; Id., «Un plagio annunciato: Girolamo Borgia e il De bello italico di Bernardo Rucellai», in C. Bastia, M. Bolognani, F. Pezzarossa (eds.), La memoria e la città. Scritture storiche tra Medioevo ed Età Moderna, Bologna, il Nove, 1995, pp. 331-360; Id., «Girolamo Borgia, Guicciardini, Machiavelli-Nifo e la caduta degli aragonesi», in V. Fera e G. Ferraù (eds.), Filologia umanistica. Per Gianvito Resta, Padova, Antenore, 1997, pp. 527-564; Id., «Preliminari per l'edizione della Historia di Girolamo Borgia», in M. de Nichilo, G. Distaso, A. Iurilli (eds.), Confini dell'Umanesimo letterario. Studi in onore di Francesco Tateo, Roma, Roma nel Rinascimento, 2002, pp. 437-466; Elena Valeri, Italia dilacerata: Girolamo Borgia nella cultura storica del Rinascimento, Milano, F. Angeli, 2007 e Mauro de Nichilo, "Hic finis pontificatus fuit”. Il Sacco di Roma nel libro XII dell'Historia di Girolamo Borgia», in A. Modigliani (ed.), Roma e il Papato nel Medioevo. Studi in onore di Massimo Miglio. II. Primi e tardi umanesimi: uomini, immagini, testi, Roma, Edizioni di Storia e Letteratura, 2012, pp. 221-231.

4. Di fatto non esistono titoli dedicati ai suoi carmina, se si escludono le rassegne delle sue opere presenti nelle pagine degli eruditi tra Sette e Ottocento, l'edizione del Carmen in triumphum Germanicum ill. Ducis B. Liviani, in Bartolomeo Cavassico, Le rime, ed. V. Cian, Bologna, Romagnoli Dall'Acqua Editore, 1893, t. I, pp. CCLXVIII-CCLXXVII (dalla trascrizione di Marin Sanudo nel cod. Venezia, Biblioteca Nazionale Marciana, Lat. XII 211) e le osservazioni, poco proficue, di Raffaele Di Florio, Girolamo Borgia poeta e storico, Salerno, stab. tip. Fratelli Jovane, 1909, pp. 41-59. 
frequentava l'accademia pontaniana, se a lui sono dedicati dal maestro versi elogiativi per una poesia elegiaca amorosa, sulla quale purtroppo non abbiamo altre notizie (Eridanus II 20,7-10) . D'altronde, dopo una prima prova militare sotto Federico, nel 1500 lo troviamo intento a seguire le lezioni di poesia del Pontano: trascriveva ex archetypis poetae ipsius una copia dell'Urania e dei Meteorum libri ${ }^{6}$, ascoltava i suoi corsi sull'Urania e su Lucrezio ${ }^{7}$, si occupava dell'educazione di Ferdinando Francesco d'Avalos, il futuro condottiero e marito di Vittoria Colonna ${ }^{8}$, I suoi versi dovettero circolare molto precocemente in quel contesto e la sua fama di poeta e seguace del gran maestro si legge nelle pagine di molti contemporanei ${ }^{9}$ : lo stato tuttavia in cui furono pubblicati i carmina dal suo discendente è tale da non permettere ad una prima lettura alcun tipo di datazione o valutazione cronologica, se non in presenza di riferimenti storici precisi, che del resto non mancano in versi di vocazione soprattutto encomiastica e celebrativa, ma soprattutto rende spesso molto difficile la stessa intelligenza del testo. Non si comprende nemmeno se i versi siano stati editi nella sequenza in cui si trovavano nei testimoni in possesso del nipote (che immaginiamo manoscritti -almeno in parte-e forse autografi) o se piuttosto egli, o il curatore materiale della raccolta, non sia intervenuto pesantemente a creare delle sezioni più o meno omogenee.

La stampa reca infatti ben poche informazioni sulle fonti utilizzate: se talvolta troviamo note come "Alias Neapoli typis excussus» ${ }^{10}$, o "Alias excussum Neapoli» ${ }^{11}$, ad indicare che si tratta di versi tratti da edizioni a stampa, manca qualsiasi indicazione sulla provenienza della maggior parte dei componimenti. É solo la necessità di menzionare coloro che gli avevano procurato nuovi testi (non lyrica né heroica in verità) a spingere il curatore ad essere più preciso. Ad un certo punto dichiara infatti di aver recuperato due epigrammi indirizzati al Cardinal Farnese «ab eruditissimis viris patre abbate Ughello et Abbate Michaeli

5. Ioannis Ioviani Pontani, Carmina, ed. B. Soldati, Firenze, G. Barbera editore, 1902, pp. 384-385: "Otia Musarum sequeris, dulcisque recessus, / Proque tuba colitur tybia blanda tibi; / Quin et amas, carmenque tibi iuvenile probatur, / Delia et ipsa tuo semper in ore sonat». Di questi versi non si dimenticherà Francesco Maria Molza in una sua elegia indirizzata allo stesso Borgia (III 6, 1-4): «Borgi, mellifluae possunt quo praeside Musae / Dura licet Stygii flectere iura Iovis, / Umbri cui licuit totiens ad carmina vatis / Inter Sirenum delituisse choros..." (Elegiae et alia, ed. M. Scorsone e R. Sodano, Torino, Res, 1999).

6. Si tratta del codice Vat. lat. 5175; cf. Benedetto Soldati, «Introduzione», in Ioannis Ioviani Pontani Carmina, cit., pp. XXXV-XXXVIII, e Mauro de Nichilo, I poemi astrologici di Giovanni Pontano. Storia del testo, Bari, Dedalo, 1975, pp. 39-56.

7. Di Lucrezio scriverà una biografia molto discussa: cf. Luigi Santo, Schede borgiane: materiale per un saggio su Gerolamo Borgia, Padova, L'altra riva, 1983, pp. 87-107 e Giuseppe Solaro, Lucrezio. Biografie umanistiche, Bari, Dedalo, 2000, pp. 31-36.

8. Cf. E. Valeri, op. cit., pp. 44-45. Roberto Zapperi, «Avalos, Francesco Ferdinando, Marchese di Pescara", in Dizionario Biografico degli Italiani, Roma, Istituto della Enciclopedia Italiana, 1962, t. IV, pp. 627-635.

9. Per una rassegna si veda M. de Nichilo, «Capitoli Borgiani», cit.

10. H. Borgiae, Carmina, cit., p. 15.

11. Ibid., p. 23. 
Iustiniano» ${ }^{12}$. Se andiamo a leggere le pagine dell'erudito Michele Giustiniani nel suo Dé Vescovi e dé governatori di Tivoli ${ }^{13}$, ci imbattiamo proprio nei due testi editi, accompagnati dalle seguenti parole:

Girolamo Borgia, Poeta celebre Napolitano del secolo passato, antico familiare di Papa Paolo III e Vescovo di Massa Lubrense (i cui componimenti poetici si trovano manoscritti presso Leone Allacci Sciotto di profonda eruditione, e dottrina greca, e latina, e Vicebibliotecario della Vaticana mio compatrioto, et amico, et i quali al presente si stampano per opera dell'erudito giureconsulto Napolitano Girolamo Borgia pronipote dell'Autore, mio amico) insinua ad Alessandro l'imitatione delle virtù dell'avo, dal quale gl'era stato dato il proprio cappello Cardinalitio, non meno, ch'il nome, con questo epigramma.

Il ruolo dell'Ughelli sembra essere stato solo quello di aver fornito le informazioni sulla presenza di un manoscritto presso Leone Allacci. Non troviamo traccia infatti degli epigrammi editi dal Borgia, né nella voce "Alexander Farnesius», del tomo secondo dell'Italia sacra ${ }^{14}$, dove pure sono trascritti due componimenti del Borgia dedicati al Cardinale, né nella voce «Hieronymus Borgius», del sesto tomo, edito nel $1659^{15}$, dove però l'erudito scriveva: "Extat hoc epigramma cum aliis plurimis ab eodem Borgio conscriptis in libello, qui nondum lucem aspexit apud doctum amicum nostrum eruditissimum Leonem Allatium». È certo che Leone Allacci, prima bibliotecario del Cardinale Francesco Barberini e poi, dal 1661, custode aggiunto della Vaticana ${ }^{16}$, avesse sotto mano l'attuale ms. Barb. lat. 1903, un apografo contenente ben 612 epigrammi, raccolti e trascritti da un solerte segretario del Borgia, oramai vescovo di Massa Lubrense e chiamato nei margini «Monsignore» ${ }^{17}$. Si può facilmente ipotizzare che tutti i componimenti editi a partire da p. 229, nell'edizione del 1666, provengano da una trascrizione dal codice dell'Allacci

12. Ibid., p. 228.

13. Michele Giustiniani, Dé vescovi e dé governatori di Tivoli libri duo, Roma, per Filippo Maria Mancini, 1665, pp. 135-136.

14. Ferdinando Ughelli, Italia sacra siue de episcopis Italiae et insularum adiacentium, rebusque ab iis praeclare gestis, deducta serie ad nostram usque aetatem, Romae, apud Bernardinum Tanum, 1647, t. II, col. 236-237.

15. F. Ughelli, op. cit., 1659, t. VI, col. 799.

16. Cf. Domenico Musti, "Allacci, Leone», in Dizionario Biografico degli Italiani, Roma, Istituto della Enciclopedia Italiana, 1960, t. II, pp. 467-471. In una lettera dell'Allacci ad Agostino Chigi, del 5 febbraio 1649 (pubblicata in Giuseppe Cugnoni, «Note al commentario della vita di Agostino Chigi», Archivio della Società Romana di Storia Patria, no 2, 1879, pp. 225226), l'erudito ricorda l'occasione in cui rinvenne il manoscritto, «dove erano da secento dodici epigrammi di Hieronimo Borgia», ovvero una ricerca fatta per conto di Luigi Novarini, intento a realizzare una raccolta antologica intitolata Martialis Christianus, mai pubblicata. In quella data l'erudito era già presso il Cardinal Barberini.

17. Presso la Biblioteca Apostolica Vaticana si conserva ancora un testimone degli epigrammi, una raccolta diversa, sicuramente antecedente a quella del Barb. Lat. 1903, e con dedica a Marcello Cervini cardinale (quindi comunque posteriore al 1539). Dal codice dell'Allacci, nel più tardo Seicento, fu trascritta una breve scelta di testi nel cod. Barb. Lat. 3231. 
fatta da Michele Giustiniani per l'amico ${ }^{18}$, dal momento che subito dopo i due epigrammi, cui si faceva riferimento supra, l'edizione dei Carmina recita:

Quae sequuntur Eiusdem Autoris Carmina habuimus a Leone Allatio viro Clarissimo et vice Bibliotecario Apostolico Eruditissimi Viri Michaelis Iustiniani beneficio.

In ogni caso una collazione tra il materiale manoscritto reperibile e la stampa non è stata mai realizzata finora e rappresenterebbe un buon punto di partenza per valutare la possibilità di mettere un ordine nelle carte del Borgia.

Ho usato l'aggettivo «reperibile» perché a livello quantitativo abbiamo oggi solo una parte, probabilmente anche esigua, dei versi che Girolamo scrisse durante tutto il percorso della sua esistenza. E non solo perché ci manca il volume di elegie che il suo segretario cita ogni tanto nei margini del Barb. lat. 1903, e di cui quindi abbiamo una precisa contezza, ma anche per quanto ci racconta un'altra fonte sempre secentesca.

Bartolomeo Chioccarello raccolse le informazioni per il suo De illustribus scriptoribus, ben prima che uscissero a stampa i Carmina del Borgia, e sicuramente a Napoli. La ricca voce bibliografica da lui compilata su Girolamo è dedicata esclusivamente alla produzione in versi, dal momento che delle Historiae dichiara di non aver trovato alcuna traccia. "Multa latino, atque optimo carmine heroico, elego, ac lyrico scripsit», dice infatti e continua: "Ex iis aliqua edita sunt, alia vero M.S. remanent; quae omnia servat Joann. Baptista de Bella Legum doctor» ${ }^{19}$. Sicuramente degno di nota è il fatto che alluda a tre diverse tipologie di carmina latini, distinte a seconda del metro usato, ovvero esametri, distici elegiaci e lirici, perché avvalora l'ipotesi che il Borgia avesse realizzato una copia in pulito dei suoi testi seguendo un criterio per genere, che -non va dimenticato- ricorda l'operazione che fu fatta sulla poesia del Pontano, al momento della pubblicazione ${ }^{20}$. E ancor più interessante è il fatto che non si soffermi assolutamente sugli epigrammi nella sua recensio (in quella data evidentemente il volume era già presso il Cardinal Barberini) e che non conosca o sembri non conoscere il pronipote del Borgia, che pure fu a lui contemporaneo.

18. I tre epigrammi editi dall'Ughelli (cf. nota 14) sono presenti nell'estratto del Barb. Vat. 3231, ma non nell'edizione del 1666. Allacci fu contattato anche per lettera dal Borgia, cf. A. Scognamiglio, op. cit., p. 289.

19. Bartolomeo Chioccarello, De illustribus scriptoribus qui in civitate et regno Neapolis ab orbe condito ad annum usque 1646 floruerunt, Neapoli, ex officina Vincentii Ursini, 1780, p. 208. Lopera fu pubblicata postuma e in parte rimane inedita: cf. Angela Casella, "Chioccarello, Bartolomeo", in Dizionario Biografico degli Italiani, Roma, Istituto della Enciclopedia Italiana, 1981, t. XXV, pp. 4-8. Su questo giurista, Giovan Battista de Bella, che, nella seconda metà del Seicento, possedeva alcune stampe e più di un codice del Borgia, non ho rinvenuto alcuna notizia.

20. Su questa questione cf. B. Soldati, op. cit. e Liliana Monti Sabia, «Una schermaglia editoriale tra Napoli e Venezia agli albori del secolo XVI», Vichiana, no 6, 1969, pp. 319-336, poi in L. Monti Sabia-S. Monti, Studi su Giovanni Pontano, Messina, Centro interdipartimentale di studi umanistici, 2010, pp. 196-214. 
Il primo item recita: «Hieronymi Borgii lecti Episcopi Massensis Solatia nostrae aetatis calamitosae ad Victoriam Columnam Heroinam illustrem», ma non viene detto se si tratta di stampa o di manoscritto. Quindi, solo dopo aver elencato alcune edizioni, fa riferimento anche ad una tradizione manoscritta, ed cita titoli che non troviamo né nella stampa del Seicento, né altrove. Un primo volume pare contenesse ben dieci egloghe, ordinate secondo uno schema vagamente virgiliano, a quanto si può con cautela ipotizzare partendo dal posto che occupano in questo elenco le poche che noi possiamo leggere oggi:

Quae autem habentur in Manuscriptis haec sunt: Ecloga I. Cynthius, Meletius, Meliseus. Ecl. II. Meletius, Meliseus, Cynthius. Ecl. III. Syrius, Damon. Ecl. IV. Felix. Ecl. V. Montanus, Chrommius. Ecl. VI Meliseus, Daphnus. Ecl. VII. Paliurus, Daphnis, Celta. Ecl. VII $<I>$. Daphnis, Paliurus, Celta. Ecl. IX. Palaemon Charopus, Menalcas. X Theogonia. Galateus, Carbo. ${ }^{21}$

In un secondo volume -anche se su questa prima suddivisione non possiamo essere sicurissimi- sono registrati ben undici componimenti, numerati dall'erudito e forse già nel suo antigrafo, di cui due soltanto editi dal Borgia ${ }^{22}$, e gli altri del tutto sconosciuti: la Magica Epiphania che nella stampa reca la dedica «ad Ferdinandum regem Catholicum» e nel manoscritto "ad Illustrem Principem D. Caesarem Aragonium Federici Regis filium», ovvero il terzo figlio di re Federico ${ }^{23}$; una Elegia, "ut in aliis codicibus legitur [così recita] Elegia Magorum» (forse una diversa redazione del primo testo); un Fons Italiae malorum; un Alexander Iracundus ad Bartholomaeum Livianum Veneti exercitus Imperatorem ${ }^{24}$; un Satyrorum lusus ${ }^{25}$, di cui vengono indicati i collocutores in «Gynopus» e «Melisaeus»; una Deploratio Hymenaei malae nuptae virginis; una Divae Constantiae Avalae Apotheosis, dedicata a Tommaso Cadamosto di Lodi, medico di Paolo III (successiva al 1541, data di morte della donna); un Panegyricus in laudem Joannis inclyti Lusitaniae Regis ${ }^{26}$; un Epitalamium Joannis Baptistae Chrysonii equitis nobilissimi; il De salutifero Christi trophaeo, e un De ortu et reparatione philosophiae ad Magnanimum Trojanum Cabanilium Montellae Regulum. Si tratta di testi appartenenti a momenti diversi e, probabilmente, tutti in verso heroicus.

21. B. Chioccarello, op. cit., p. 209.

22. Nel volume del 1666 troviamo a stampa la Magorum Epiphania (pp. 40-49) e il De salutifero Christi trophaeo, con dedica ai Padri Teatini (pp. 82-89).

23. Il principe morì a soli diciotto anni nel 1520: è possibile che la dedica gli fosse stata indirizzata quando era ancora bambino; in tal caso la redazione intitolata a Ferdinando il Cattolico (morto nel 1516) potrebbe essere successiva. Ma siamo nel campo delle mere ipotesi.

24. Si tratta probabilmente di un altro panegirico del condottiero, con un titolo che sembra riecheggiare quello del più famoso Orlando furioso dell'Ariosto, se non fosse impossibile una datazione posteriore al 1516 (prima edizione del poema), dal momento che l'Aviano morì nel 1515 .

25. Il testo recita Satyrarum, ma credo reclami questa correzione.

26. Anche il Britonio (sul quale cf. nota 46) scrive un panegirico indirizzato a Giacomo III di Portogallo: cf. Eugenio Asensio, "El italiano Britonio, cantor de la "Lisboa de D. Joâo III"”, Arquivos do Centro Cultural Português, no 5, 1972, pp. 546-559. 
"In alio Manuscripto codice operum eiusdem Hieronymi Borgii Poemata ac elegiaen: dice poi il Chioccarello, e cita in ordine un Romae excidium, ovvero una elegia sul sacco del 1527, un carmen Ad Urbem infelicem, per l'esondazione del Tevere del 1530, il Panegyricus de inclita victoria Bartholomaei exercitus Imperatoris contra Germanos (1508?), una Apologia in favore dello stesso indirizzata a Pietro Bembo e datata al 1513, un De pugna Ravennate (1512?), ancora un Panegyricus de laudibus inclitae Heroinae Isabellae Aragoniae indirizzata a Cristoforo Colonna (anteriore al 1533?)27, una Tragica elegia ad Italiam et Galliam infelices, una Egloga Gallicana indirizzata a "Joannes Meletus gallus discipulus suus», seguita da un'egloga Daphe (sc. Daphne), «in qua Franciscus Petrarca et Laurus manu ipsius apud tumulum in agro Patavino consita praecipue canuntun, e ancora una non altrimenti specificata Elegia. "Varia etiam epigrammata, Hymni, aliaque carmina in iis manuscriptis libris habentun, aggiunge a concludere la descrizione.

L'unico commento riguarda il Fons Italiae malorum ed è teso a sottolineare la vocazione civile di molti di questi componimenti:

Ex aliquibus ipsius manuscriptis operibus, et praesertim ex eo poemate quod inscribitur Fons Italiae malorum, habes eius temporis miserias, ac calamitates, et quidquid boni, vel mali in Regno hoc, aliisque Italiae locis citat. Is autem, sub fictis Satyrorum ac pastorum nominibus, principes viros, nationes, aliosque recenset, ac carpit.

Se ci fermiamo ad osservare l'elenco di testi «bucolici» e lo confrontiamo con quello che noi possediamo e leggiamo del Borgia oggi, ci rendiamo conto che i dati raccolti dal Chioccarello, di seconda mano e forse parzialmente inesatti, non possono che essere tuttavia fededegni: altrimenti non avremmo alcune curiose ripetizioni e alcune descrizioni accurate che ci tornano molto utili per la lettura che ci apprestiamo a fare delle egloghe sopravvissute. A noi mancano molti testi "pastorali»: sei egloghe delle dieci elencate, il Lusus Satyrorum e infine il Fons Italiae malorum, che sotto il velo dell'allegoria cantava i mali dell'Italia contemporanea ${ }^{28}$. Almeno dodici carmina bucolica aveva scritto dunque Girolamo e credo sia lecito sospettare anche che avesse pensato ad un Bucolicum carmen, magari mai sistemato in via definitiva.

In questa sede la mia attenzione sarà dedicata esclusivamente al primo testo recensito dal Chioccarello, che nell'edizione reca il titolo variante Solatia aetatis calamitosae, e in particolare -per ragioni di spazio- al primo dei due

27. Cristoforo o Crisostomo Colonna fu precettore di Ferdinando d'Aragona, fratello di Cesare e figlio di Isabella del Balzo e di Federico I; a lui Antonio Galateo indirizza la sua lettera $D e$ educatione (cf. Antonio De Ferrariis, De educatione. 1505, ed. C. Vecce, P. Tordeur, P. Jodogne, Bruxelles, Peeters Press, 1993). Fu lui ad essere chiamato inoltre come precettore di Bona Sforza: cf. Carlo Vecce, «Il De educatione di Antonio Galateo De Ferrariis», Studi e problemi di critica testuale, no 36, 1988, pp. 23-82 e Domenico Defilippis, Tradizione umanistica e cultura nobiliare nell'opera di Belisario Acquaviva. Gli opuscoli pedagogici del Conte di Conversano e Duca di Nardò, Galatina, Congedo, 1994, pp. 48-53.

28. Da questa descrizione si potrebbe anche sospettare che si tratti anche in questo caso di un Satyrorum lusus. 
componimenti. Dedicati a Vittoria Colonna, i Solatia comprendono di fatto le ecloghe I e III dell'elenco di dieci riportato nel De illustribus scriptoribus: ma entrambe sono ricordate anche nella recensio del terzo codice compulsato dal Chioccarello, quello delle Elegiae, col titolo di Gallicana e Daphne, situate subito dopo la Tragica elegia ad Italiam et Galliam infelices. È possibile dunque -se è lecito fare ipotesi- che originariamente fossero legate insieme, e che in seguito il Borgia le abbia ricollocate diversamente in una struttura più ampia. Ma qui il discorso si muove entro i confini nebbiosi del territorio della filologia secentesca, che lasciano molta libertà alla fantasia del moderno studioso e pochissimi dati certi.

Lordine in cui i componimenti bucolici compaiono nell'edizione è ancora diverso, ovvero: Theogonia (la decima nella lista del Chioccarello), Felix e Solatia. Ma nella farragine secentesca i tre testi (dal momento che i Solatia rappresentano un corpo unico) sono incastonati in una struttura estremamente artificiosa, che li separa e li unisce allo stesso tempo: ogni egloga è inserita all'interno di due gruppi di distica, intervallati da odi oraziane. La varietas così ottenuta (che rispecchia l'inscriptio del volume, "carmina lyrica et heroica») è molto di maniera e dubito che possa risalire all'autore. Quello che sembra emergere comunque da questa composizione non è sicuramente un ordine cronologico quanto invece l'idea che i tre testi, avvicinati sicuramente pensando al genus bucolicum, non provenissero da un antigrafo contenente un Bucolicum carmen di dieci egloghe, che altrimenti sarebbero state pubblicate tutte, a nostro avviso.

Una veloce lettura dei primi due, Theogonia e Felix ${ }^{29}$, sui quali non posso fermarmi in questa occasione, ci mostra l'autore perfettamente a suo agio in quella varietas di toni bucolici, che, agli inizi del Cinquecento, declinava il genere in forme altamente raffinate e differenziate, contemplando sia il manierismo lirico (penso, in ambito ancora napoletano, ai versi di Pomponio Gaurico) $)^{30}$ sia ancora l'allegorismo politico, sia più spesso la vocazione encomiastica, commemorativa e autobiografica. La prima egloga, in ordine di stampa, Theogonia, rientra in quel filone di poesia bucolica sacra, inaugurato da Francesco Patrizi negli anni quaranta del Quattrocento e proseguito in forme diverse per tutto il secolo e oltre ${ }^{31}$ : due pastori dai nomi decisamente

29. L'egloga Felix, ricordata in quarta posizione nella lista delle dieci, si conserva in una redazione leggermente diversa in un manoscritto della biblioteca del Colocci, il Vat. lat. 5225; mentre quella intitolata Theogonia, la decima, la leggiamo solo nell'edizione secentesca.

30. Cf. Pompeo Giannantonio, «Pomponio Gaurico, gli umanisti napoletani e la corte aragonese», in I Gaurico e il Rinascimento, cit., pp. 97-108 e Giovanni Ponte, «Le egloghe di Pomponio Gaurico fra stilizzazione letteraria e sperimentalismo», ibid., pp. 247-256.

31. Oltre all'oramai datato Giulia Calisti, Il De partu Virginis di Jacopo Sannazzaro. Saggio sul poema sacro nel Rinascimento, Città di Castello, «Il Solco», 1926 rimando a Claudia Corfiati, "Note sulla poesia bucolica di Egidio da Viterbo», in Egidio da Viterbo, cardinale agostiniano, tra Roma e l'Europa nel Rinascimento, Atti del Convegno di studi, Roma, 26-28 settembre 2012, Roma, Roma nel Rinascimento, 2014, pp. 381-402. 
accademici, Galateo e Carbone ${ }^{32}$, rievocano, l'uno in prima persona, l'altro ricordando il canto di un terzo pastore, l'evento della nascita di Cristo. L'Egloga Felix invece è stata probabilmente concepita durante uno dei soggiorni romani del Borgia per esaltare Felice Della Rovere, figlia di papa Giulio II e andata sposa a Giovanni Giordano Orsini: Febo, circondato dalle Muse, da Pallade, da Venere e dalle Grazie intona all'ombra di una "quercia» un canto di lode nei confronti di questa «nympha Felix» e poi del genitore Iulius, chiamato «magnanimus pastor».

Le due egloghe indirizzate a Vittoria Colonna invece, un vero e proprio dittico bucolico, circolato sotto il titolo di Solatia aetatis calamitosae, si distinguono per una forte ascendenza petrarchesca e direi anche sannazariana, per la struttura drammatica quasi identica, nonché per un'ispirazione che oserei dire unitaria. La datazione di questo dittico, come negli altri due casi, è molto difficile. Sicuramente appartengono al periodo napoletano del Borgia, e fanno riferimento entrambe a una situazione di calamitas, non altrimenti specificata.

Analizziamo dunque la prima, Gallicana secondo una parte della tradizione. Essa contiene una sezione che potremmo definire introduttiva rispetto ad entrambi i componimenti, in cui la Colonna viene chiamata in causa quasi come una Musa ispiratrice, una "Nympha Iunionia», pronta ad ascoltare i versi pastorali del poeta, ispirati dall'onda di un «noster Mincius», cui, con il permesso di Aretusa, attinge, nel tentativo di portare conforto in un momento di profonda angoscia e paura (dice infatti, con un plurale che non credo sia maiestatis, ma alluda ad un contesto di intellettuali ben preciso, "nos attoniti»), in presenza di un "dirus tumultus minitans", e di una "rabida discordia" che sommuove "insani reges», folli regnanti che si accaniscono in una guerra ormai stanca. Dopo l'invocazione alla Colonna seguono alcuni versi che ripetono un modulo tipico del proemio epico con l'indicazione del contenuto del poema: Borgia canterà l'arrivo di due pastori e la causa del loro lungo girovagare.

\footnotetext{
Pastorum adventum longinquarumque viarum dicemus causam (Gallorum horrenda ferentur ${ }^{33}$ ostenta et diris ferientur vocibus aures), qui postquam variis defuncti erroribus oras Sirenum cultas videre. ${ }^{34}$
}

32. Si tratta di due personaggi entrambi attivi ai tempi d'oro dell'Accademia e probabilmente ancora vivi all'epoca di composizione di quest'egloga: Antonio De Ferrariis detto il Galateo e il meno noto Girolamo Carbone (su cui cf. Pierre de Montera, L'humaniste Napolitain Girolamo Carbone et ses poésies inédites, Napoli, Riccardo Ricciardi Editore, 1935; Renato Pastore, "Carbone, Girolamo", in Dizionario Biografico degli Italiani, Roma, Istituto della Enciclopedia Italiana, 1976, t. XIX, pp. 695-698; Antonietta Iacono, «Sull'elegia di Girolamo Carbone ad Auroram puellam», in G. Catanzaro e F. Santucci (eds.), Poesia umanistica latina in distici elegiaci, Assisi, Accademia Properziana del Subasio, 1999, pp. 303-324).

33. La lezione della stampa è ferentam, e anche dopo sembra leggersi un ferient ve invece del più corretto ferientur: si noti la paronomasia. In questo contesto non posso soffermarmi sul testo dell'egloga, che reclama seri interventi di emendatio in più luoghi. Auspico di poter pubblicare criticamente i Solatia in altra sede.

34. H. Borgiae, Carmina, cit., p. 185. 
La leggera suspense creata da questo incipit, si mantiene viva per buona parte dell'egloga. I due pastori in questione, chiamati Cintius e Meletius, sono giunti sulle coste campane, le cultae orae Sirenum, dopo aver errato per molto tempo. Assomigliano in questo a pellegrini dunque, che stanchi approdano in un luogo familiare -e questo lo si intuisce fin da subito- alla ricerca di qualcuno o di qualcosa. Cinzio, personaggio dietro il quale si nasconde-come vedremo- l'autore stesso, riconosce i luoghi di Napoli a lui cari, i foelices colles, l'aura maris, e le voces canorae Sirenum, che rimandano non solo al mito della sirena Partenope sulla cui tomba fu fondata la città, ma ad un topos molto caro a tutta la poesia napoletana contemporanea, e al Pontano in particolare. Solo a questo punto, siamo al v. 36, riceviamo la notizia che si tratta delle patriae orae: «O quid amabilius patria?», esclama infatti. E immediatamente dopo ci viene rivelato il luogo preciso in cui i due pastori erranti sono approdati, ovvero Antiniana, la campagna del Pontano, il «rus Melisaei». Cinzio conosce bene quei giardini ed indica al suo compagno di viaggio gli alberi di alloro sulla cui corteccia ancora si vede scritto il nome di Ariadna, la cui morte è cantata dal grande umanista nella sua seconda egloga. Segue una elencazione di alcune opere poetiche del Pontano, evocate da immagini che le rappresentano in vario modo: la Lyra, l'Urania, considerata l'impresa maggiore e quella in cui si esprime una piena maturità stilistica e infine il De hortis Hesperidum, l'ultima fatica del poeta, nascosto sotto l'ombra di un citrus, un cedro ${ }^{35}$.

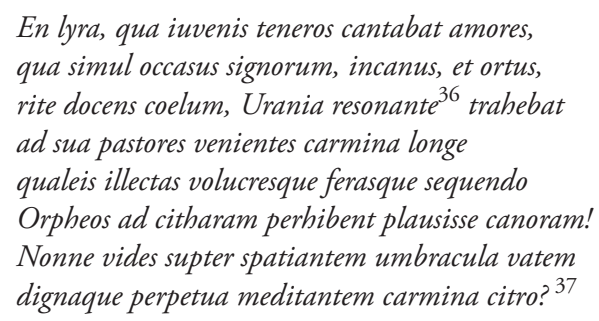

Melezio è invitato ad inginocchiarsi davanti al vecchio Pontano e a comunicargli le ragioni del loro viaggio. Nell'ultimo verso di questo primo intervento di Cinzio compare l'accenno ad una figura del resto muta, un "Epytides Briton», che "promet munuscula», un personaggio dunque, che nel patronimico rimanda alla figura di Perifante, il precettore di Ascanio in Aen. $\mathrm{V} 545$, e che porta con sé dei doni. Il breve intervento del giovane Melezio è anch'esso molto epico: si rivolge al vecchio poeta chiamandolo «magne paten»,

35. Sul De hortis Hesperidum, ultima fatica del Pontano, cf. Bruno Figliuolo, «Nuovi documenti sulla datazione del De hortis Hesperidum di Giovanni Pontano", Studi Rinascimentali, no 7, 2009, pp. 11-15 e Antonietta Iacono, «Il De hortis Hesperidum tra innovazioni umanistiche e tradizione classica», Spolia. Journal of Medieval Studies, vol. I, 2015, pp. 188-237.

36. La lezione della stampa è res ante.

37. H. Borgiae, Carmina, cit., p. 186. 
attributo che nel mondo classico si trova nelle preghiere rivolte a Giove, e gli spiega di essere giunto a Napoli «iussu Celtarum pastorum» per chiedere a lui un responso.

$$
\begin{aligned}
& \text { Magne pater, cuius nostri penetravit ad undas } \\
& \text { Oceani et Morinos, etiam tibi, fama, repostos } \\
& \text { iunxit amore pio, superatis alpibus et tot } \\
& \text { terrarum spatia emensi, tot flumina vasta, } \\
& \text { Caeltarum iussu pastorum venimus ad te } \\
& \text { certa tua in dubiis scitatum oracula rebus. } \\
& \text { Te petimus, penitus fatemur arcana tenentem } \\
& \text { quemque superstitio popularis nulla fatigat. }
\end{aligned}
$$

Il lettore si rende conto che il vecchio Meliseo ha acquistato, in questa sua trasfigurazione bucolica, il ruolo della Sibilla cumana, le capacità profetiche che solo un vate "politico" può far credere al mondo di possedere. La sua auctoritas è punto di riferimento in dubiis rebus, ma non si tratta di una forma di «magia» o di «religiosità pagana», dal momento che la superstitio non appartiene in alcun modo al poeta. Meletius, dietro il quale si nasconde un ragazzo di cui il Borgia fu probabilmente precettore (il nome latinizzato potrebbe nascondere un Jean Mellet o Melet) ha ricevuto l'incarico dai Celtae (indicazione generica che potrebbe rimandare alla Gallia e quindi alla Francia) di compiere un viaggio, simile a quello di Enea in Campania, per conoscere il responso del grande maestro. Questo giovane risulta l'autore di un opuscolo, stampato a Roma il 28 febbraio del 1525, la cui epigrafe recita: «Ioanni Meletii Italogalli Reip. Christianae et Italicae libertatis amatoris Tragica elegia ad Italiam et Galliam infelices», ovvero «Elegia tragica indirizzata a Italia e Francia infelici dell'italofrancese e amante della cristianità e della libertà d'Italia Jean Melet». Si tratta effettivamente di una lunga elegia, seguita da un breve testo Ad Regis matrem, che nasce come lamento della Francia dopo la sconfitta di Francesco I nella battaglia di Pavia ${ }^{39}$ : l'avvenimento avrebbe dovuto rappresentare uno sprone per una pacificazione e per una liberazione dell'Italia dalle guerre, secondo l'autore dei versi. Questo testo d'occasione però, la cui circolazione dovette essere minima e ristretta, ci interessa perché è in verità tra quelli recensiti dal Chioccarello come opera del Borgia, e presente in uno dei manoscritti da lui compulsati, anzi viene citato immediatamente prima dell'egloga Gallicana, cioè il testo che stiamo commentando, ovvero la prima egloga dei Solatia. Ė altamente probabile che, pur pubblicato sotto il nome del discepolo, esso sia stato scritto dal Borgia stesso e conservato tra le

38. H. Borgiae, Carmina, cit., p. 186.

39. Su questa battaglia, su cui molto si è scritto, rimando al recente intervento di Paolo Pintacuda, «La battaglia di Pavia nella letteratura spagnola dei secoli d'oro», in Rendiconti dell'Istituto Lombardo di scienze e lettere, vol. 130, 1995, pp. 395-427 e alla bibliografia ivi citata. L'esito della vicenda fu variamente celebrato nell'arte e nella letteratura; ricordo un breve testo, teatrale e pastorale, spagnolo -cf. Joseph Eugene Gillet, "A Spanish Play on the Battle of Pavia (1525)", in Proceedings of the Modern Language Association, no 45, 1930, pp. 516-531- o ancora la menzione dell'episodio nell'Orlando Furioso del 1532 (cf. Angelo Cerri, «L'Ariosto e la Battaglia di Pavia», in Giornale Storico della Letteratura Italiana, no 152, 1975, pp. 551-556). 
sue carte insieme a quei versi bucolici che l'erudito secentesco diceva dedicati proprio al Melet. Inoltre, come si è detto, dal momento che il testo a stampa dell'egloga -non possiamo certo divinare sulla redazione manoscritta- vede il giovane Meletius tra i protagonisti, la Tragica elegia potrebbe essere utile per la datazione almeno della prima egloga dei Solatia.

Dopo la richiesta di un responso espressa dal ragazzo dunque, il Pontano, che nella sua vecchiaia è diventato anche un po' sordo, chiede qualche informazione di più ai due iuvenes che gli si sono presentati, usando ancora parole virgiliane, che ricordano la scena del libro VIII dell'Eneide (vv. 112-114), in cui Enea raggiunge le terre di Evandro e questi, vedendolo arrivare con i suoi uomini, prima di riconoscere in lui il figlio del suo caro amico Anchise, gli chiede:

\author{
"iuvenes, quae causa subegit \\ Ignotas temptare vias? quo tenditis?", inquit. \\ "Qui genus? unde domo? pacemne huc fertis an arma?"
}

Questo è un luogo importante nella memoria poetica di questa egloga, insieme a quello corrispondente dell'approdo di Enea sulle coste campane: in entrambi i casi si tratta dell'incontro di eroi esuli, sfuggiti da un conflitto concluso o meno, che cercano nell'ospite anziano accoglienza e un giusto consiglio per proseguire il proprio percorso. Il vecchio poeta dunque vuole sapere chi sono, ma chiede anche al ragazzo di parlare più forte: "Dicite rursus, et herculeam de gutture vocem / promite, surda senis clamoribus indiget auris» ${ }^{40}$. $\mathrm{Ma}$ ancor prima di sentire la risposta si accorge che Cinzio non gli è del tutto estraneo, anzi lo riconosce come suo discepolo, colui a cui ha insegnato un carmen, che iniziava "Qui coelo radient ignes [è il primo verso dell'Urania] qui spiritus auras / sollicitet [è il primo verso, secondo emistichio, dei Meteora]»; ma Pontano si ricordava un ragazzo biondo, ora invece $c a n e t^{41}$. Cinzio risponde che sono state le curae e il labor che lo hanno fatto incanutire prima del tempo: egli, il Borgia, si presenta qui come un nuovo Melibeo, costretto ad errare, e che «invidia» coloro cui la sorte ha concesso di restare in patria, a Napoli, Anisio prima di tutto, «nimium felix», e Summonte. Il motivo per cui egli si è allontanato tuttavia non è l'esproprio delle terre o la guerra, ma -almeno così confessa in questo punto- l'amor per le Camene externae, ovvero -non saprei intendere diversamente- il desiderio di un perfezionamento nella letteratura greca, che lo ha portato a frequentare, stultus, gli accampamenti stranieri (se vogliamo tradurre alla lettera quel castra, ma qui probabilmente va inteso in senso allusivo). Sappiamo bene che Girolamo, quando si allontanò da Napoli frequentò anche l'università di Padova e Venezia, dove perfezionò la sua conoscenza del greco, e soprattutto la così detta Accademia Liviana ${ }^{42}$. Cinzio

40. H. Borgiae, Carmina, cit., p. 186.

41. Al di là del motivo autobiografico, che pure ha una sua importanza, non va dimenticato che il poeta pastorale, da Titiro in poi, rappresenta la sua dura esperienza di vita con la canizie.

42. Renata Fabbri, "Bartolomeo d'Alviano, condottiero e mecenate, e l'Accademia Liviana”", in La Serenissima e il Regno nel V centenario dell'Arcadia di Iacopo Sannazaro (Bari, 4-5 ottobreVenezia, 7-8 ottobre 2004), Atti del Convegno di studi, Bari, Cacucci Editore, 2006, pp. 265-284. 
dice inoltre di essere stato in Gallia, ovvero nel Nord Italia, ben tre lustri, quindici anni, e che sono stati i «dites pastores» che lo hanno spedito in patria a consultare il Pontano. Ribadisce qui che sono in tre: lui, un suo discepolo («noster alumnus») e questo Epitide, che è un bravo suonatore di canne.

Svelata, per dir così, l'identità del piccolo gruppo di pastori erranti, Meliseo li accoglie amorevolmente, li fa accomodare ai piedi del suo alloro, offre loro dei frutti dolci provenienti dall'Oriente, delle pesche, e del vino, li invita al sonno, rimandando al giorno successivo la conversazione ${ }^{43}$. Questi versi non sono un semplice richiamo alla tradizionale ospitalità dei pastori bucolici (a partire dalla prima egloga di Virgilio in poi si potrebbero fare numerosissimi esempi in proposito), ma -a mio parere- implicano una allusione ben più importante all'Aegidius del Pontano. Come è noto infatti anche in quel contesto, apparentemente lontanissimo dal genus bucolicum, vi sono due pellegrini (di cui uno sicuramente proviene dal Nord Italia, Suardino Suardo) che giungono a Napoli e si avvicinano alla dimora del gran Pontano, che riconoscono da una certa fisionomia e dal modo di parlare, e al quale si presentano stanchi e polverosi, provenendo dalle campagne infestate dalla guerra. L'autore li fa accomodare, offre loro della frutta fresca e del vino e poi inizia la lunga conversazione accademica che vedrà intervenire altri personaggi (tra cui anche il Summonte). Poco più tardi nel dialogo, il Pontano terrà una lezione sugli oracoli e sulla possibilità che Dio si serva di menti ispirate come tramite per comunicare ai mortali messaggi di particolare importanza. Non solo, ma non va dimenticata, sempre nelle pagine del dialogo, l'evocazione del fantasma di Gabriele Altilio. Cos'è d'altronde in questo contesto bucolico Melisaeus se non il fantasma del Pontano? Dal momento infatti che il Borgia partì per il Nord Italia dopo la morte del maestro, tornando dopo quindici anni non può raccontarci di averlo incontrato sensa provocare nel lettore un senso di straniamento: operazione giustificabile solo se pensiamo al culto quasi religioso per il maestro, vivissimo ancora negli anni in cui questa egloga fu scritta. Se l'egloga Melisaeus di Giano Anisio ${ }^{44}$ viene ascritta da Carlo Vecce ad un tempo non molto posteriore alla morte del Pontano, per la presenza di Cotta, compagno di viaggi del Borgia e anche lui frequentatore dell'Accademia liviana, morto a soli trenta anni nel 1510, non dobbiamo dimenticare che l'Arcadia del Sannazaro, edita nel 1504, si chiudeva con una "comparsa» di Meliseo e che anni più tardi, probabilmente poco prima dell'esperimento borgiano, Basilio Zanchi dedicava alla scomparsa di Melisaeus una lunga bucolica monodica ${ }^{45}$,

43. Questo fatto rende plausibile l'esistenza di una seconda egloga con gli stessi personaggi, come risultava dall'elenco del Chioccarello. D'altronde nella lista delle dieci egloghe anche la settima e l'ottava hanno gli stessi personaggi: l'uso di dividere in due episodi la fabula pastorale era stato avviato da Petrarca (con le due egloghe contro il papato avignonese) e perseguito da altri umanisti nel Quattrocento (penso ad esempio alle prime due egloghe di Tito Vespasiano Strozzi).

44. Carlo Vecce, «L'Egloga Melisaeus di Giano Anisio tra Pontano e Sannazaro», in Stefano Carrai (ed.), La poesia pastorale nel Rinascimento, Padova, Antenore, 1998, pp. 213-234.

45. Lawrence V. Ryan, «Milton's Epitaphium Damonis and B. Zanchi's Elegy on Baldassare 
conservata anche nella farragine di poesia cinquecentesca contenuta nel vol. IV del ms. Vat. lat. 5225 (cc. 838v-842v) con il titolo Amilcon a quo Joviani Pontani mors deploratur, a poche carte di distanza dall'egloga Felix del Borgia.

Dopo aver dunque consigliato ai tre ospiti di riposare, il vecchio aggiunge un vero e proprio incitamento al sonno, sicchè il lettore si aspetterebbe un explicit. Invece il discorso del Pontano continua: invita alcuni pueri ad aprire le fonti per una terra assetata e quindi, se dovessimo attenerci ad una interpretazione letterale, ad irrigare i campi, e recita un vero e proprio encomio dell'agricoltura, dell'allevamento e dell'apicultura (con chiaro rimando ai libri delle Georgiche). Non possiamo però leggere questi versi come puro ornamento poetico, finalizzato a delineare il ritratto di un Pontano pastor o meglio colonus (maschera con cui lui stesso aveva giocato nell'Asinus): in essi credo vada riconosciuta l'immagine allegorica del lavoro intellettuale (già utilizzata dal Petrarca nella sua nona egloga), un labor che assume le caratteristiche di bonus e sanctus ("nil agri melius cultu / nil sanctius»), di uber e dulcis ("nil uberius pecoris nil dulcius arte tuendi humano generi dii concessere labores»).

Ma il suo discorso viene interrotto dall'apparizione improvvisa di una cometa, visione infausta: "numquam arsit sine vasta animantum clade cometes», sentenzia infatti. I versi che seguono sono una vera e propria profezia, costruita secondo moduli ben sperimentati dalla poesia bucolica umanistica, su imitazione della famosa quarta egloga virgiliana, non senza l'influenza del profetismo cinquecentesco. La visione - perché viene presentata in questi termini, con il verbo cerno in posizione forte alla fine del primo verso- è terrificante. Branchi di bestie sono diretti in una corsa sfrenata contro i nostra vivaria, termine con il quale sicuramente si riferisce ai culti agri della Campania: si tratta di bestie barbariche che a breve invaderanno tutta l'Italia. Ecco che lui chiama i pastores alle armi, li invita a provvedere meglio alla difesa dei recinti, ma davanti ai suoi occhi queste bestie feroci si avvicinano: si tratta di un leone, di un immane orso e di un serpente, il più difficile da evitare ${ }^{46}$. Questi tre mostri non possono che essere sconfitti con l'aiuto degli dei, per cui è necessario un sacrificio, offrire una vitella, placare l'ira divina, pentirsi dei peccati. È invocato quindi Pan, che qui -diversamente dalle abitudini della poesia bucolica neolatina- è chiaramente trasfigurazione di Cristo:

Pan, o magne, piis quem ploravere peremptum
vocibus in caelo volucres et in aequore nymphae
quem trunco in sacro nimium crudelia passum
supplicia ante alios lacrymis sol flevit abortis
cum caput obscuro nitidum praetexit amictu
indignumque nefas simul adversatus anhelos
retro flexit equos tenebrisque exterruit orbem
unde sibi aeternam timuit gens impia noctem.

Castiglione», Humanistica Lovaniensia, no 30, 1981, pp. 108-117.

46. Non mi cimento per il momento in una lettura di queste figurae, dietro le quali sicuramente si nascondono i soggetti politici più ostili, dal punto di vista del Borgia, alla pace in Italia. 
Exoptant alii longam bene vivere vitam, ast ego sat bene posse mori, da lumina claudam ne videam terris quod cunctis imminet ingens diluvium agrestemque casas regumque superbas mersurum passim turres ac templa deorum. ${ }^{47}$

Pontano desidera chiudere gli occhi prima di assistere a quello che per lui è un nuovo diluvio universale, che spazzerà via tutto sommergendo poveri, ricchi, i palazzi e le chiese. La conclusione di questo testo è decisamente apocalittica, con un'immagine molto forte, una visione di un futuro che naturalmente è insieme il presente e il passato prossimo, che Borgia ha vissuto con quel sentimento di sofferente impotenza che tutti gli intellettuali napoletani, pur di diverse generazioni, furono costretti a provare davanti all'accanirsi di una situazione di conflitto tra Francia e Spagna durato quasi un trentennio.

L'indicazione dei tre lustri, ovvero di quindici anni di esilio, potrebbe in prima battuta suggerire una datazione al 1519, dal momento che la partenza per il Nord Italia al seguito di Bartolomeo d'Alviano risale effettivamente al 1504. A questo anno ci riporterebbe anche la menzione del Britonio -se è lui il Briton che accompagna silenzioso i due pellegrini-, poeta all'epoca fortemente legato alla Colonna, tanto da dedicarle la sua prima raccolta poetica, $\mathrm{La}$ gelosia del Sole ${ }^{48}$. Elena Valeri ha recentemente ribadito invece la datazione dei Solatia al 1528, già avanzata dalla Giordano ${ }^{49}$, interpretando l'atmosfera quasi tragica che viene evocata nei primi versi e poi ripresa all'interno delle egloghe con quella che sicuramente si respirò a Napoli, e ancor più a Ischia, durante l'anno di assedio della città da parte dei francesi. Credo tuttavia improbabile che Borgia avrebbe accolto il giovane italofrancese Melet tra i personaggi dell'egloga in quel contesto.

Se proviamo invece a sommare i quindici anni non alla data della prima partenza del Borgia, ma alla data della seconda, ossia al 1510, e ad accostare la profezia di Meliseo alle parole iniziali della Tragica elegia attribuita al Melet, rimaniamo sorpresi da una forte sintonia. Nel 1525 la interrogazione di un gallus sulle sorti dell'Italia avrebbe avuto non solo senso, ma una piena giustificazione: Francesco I infatti era stato fatto prigioniero a Pavia, non si conoscevano ancora le sorti del regno e le scelte che avrebbe fatto il pontefice, e inoltre circolava da anni una profezia che annunciava per quella data, a causa di una particolare congiunzione astrale nel segno dei Pesci, un diluvio universale. Tutte queste circostanze potrebbero aver suggerito al Borgia, sempre attentissimo ai fenomeni politici, un testo d'occasione come la Tragica elegia, e un più elaborato, perché scritto in e rivolto ad un contesto molto più specifico

47. H. Borgiae, Carmina, cit., pp. 188-189.

48. Su di lui cf. Gianni Ballistrieri, "Britonio, Girolamo», in Dizionario Biografico degli Italiani, Roma, Istituto della Enciclopedia Italiana, 1972, t. XIV, pp. 347-349; si veda ora Girolamo Britonio, La gelosia del sole, a cura di M. Marrocco, Roma, Sapienza Università editrice, 2016, e bibliografia ivi citata.

49. Amalia Giordano, La dimora di Vittoria Colonna a Napoli, Napoli, Tipografia Melfi \& Joele, 1906, p. 80. Cf. E. Valeri, op. cit., pp. 78-79. 
quale il circolo di intellettuali raccolti intorno alla Colonna, carmen bucolicum. Non solo nell'elegia vi è un riferimento a viri fatidici che avevano previsto l'esito tragico di una vicenda bellica che si trascinava da anni (allusione alle profezie ritorna in maniera differente nella figura fatidica di Melisaeus), ma anche a quel «diluvio» che avrebbe travolto tutto e tutti ${ }^{50}$ :

Eripe musa oculis nubem mortalibus atram
ac rumpe horribili pectora dura sono,
Obruta diluvio est infelix Gallia vasto
dira quid in terras saevius astra pluent.
Quam timeo haec orbem ne fluctibus obruat omnem
tempestas populos sanguinolenta necans.

Certo vi è uno scarto notevole tra i due messaggi, sicuramente legato all'immediatezza del primo (scritto pochi giorni dopo l'esito della battaglia) e alla più ampia invece prospettiva del secondo, che guarda all'aspetto politico solo marginalmente, innestandolo su di una nostalgica rievocazione delle glorie culturali della Napoli aragonese. Solo una verifica dei testi e un confronto puntuale con le fonti storiche coeve, potranno permettere in altra sede di approfondire e corroborare questa ipotesi.

Possiamo solo aggiungere che la presenza della Colonna ad Ischia è ben documentata negli anni dal 1517 al $1525^{52}$, anno di morte del marito Ferrante proprio in seguito alle ferite riportate durante la battaglia di Pavia nella quale si distinse dalla parte dell'Imperatore, e che in quello stesso 1525 il Britonio pubblicava a Napoli un Triompho nel quale Parthenope sirena narra et canta gli gloriosi gesti del gran marchese di Pescara, e il Borgia a Roma la Ad Carolum Caesarem opt. max. Monarchia, dedicato a Luis Hernandez de Cordoba, ambasciatore a Roma, opuscolo che si conclude con una Victoria Caesarea Armipotentis Ferrandi Avali Neapolitani Magni Marchionis Piscariae Caesarei Ducis de Francisco Gallorum Rege Maximo apud Ticinum die memorabili XXV Februarii MDXXV. È un momento di grave lutto per la Colonna e nello stesso tempo di necessaria, profonda riflessione sugli assurdi esiti di un conflitto che si protraeva da molto tempo. Da questo punto di vista la seconda egloga, che apparentemente non ci fornisce alcun aiuto per la datazione, nell'accostare alla

50. Sul profetismo estremamente fecondo in questi anni, e in particolare sul pronostico del diluvio e sulle visioni dei così detti eserciti furiosi si veda: Ottavia Niccoli, Profeti e popolo nell'Italia del Cinquecento, Roma, Laterza, 1987. È notevole che vi sia l'evocazione di un vates fatidicus anche nel IX libro delle Historiae del Borgia: cf. E. Valeri, op. cit., p. 45.

51. Ioanni Meletii Tragica elegia ad Italiam et Galliam infelices, Romae, s. n., die XXVIII Feb. M. D. XXV, c. Aiiv.

52. La bibliografia sulla poetessa è assai vasta, per quello che ci interessa in questa sede cf. oltre a A. Giordano, op. cit., Suzanne Therault, Un Cénacle humaniste de la Renaissance autour de Vittoria Colonna châtelaine d'Ischia, Firenze-Parigi, Sansoni-Didier, 1968, Giorgio Patrizi, "Colonna, Vittoria», in Dizionario Biografico degli Italiani, Roma, Istituto della Enciclopedia Italiana, 1982, t. XXVII, pp. 448-457 e Tobia R. Toscano, Letterati corti accademie. La letteratura a Napoli nella prima metà del Cinquecento, Napoli, Loffredo, 2000, in part. pp. 13-24. Non si dimentichi che il Borgia dedica a Vittoria il X libro delle sue Historiae. 
leggenda del Pontano un altro personaggio che in quegli anni dominava la cultura letteraria italiana ed europea, e sicuramente molto caro alla Colonna, ovvero Petrarca, ci suggerisce di leggere il dittico come un tentativo di descrivere la cultura napoletana dei primi decenni del Cinquecento, nella sua duplice ascendenza, latina e pontaniana da una parte (non scevra da un residuale afflato politico), volgare e petrarchesca dall'altra: entrambe queste anime si incontravano intorno a Vittoria Colonna e il Borgia cerca di rappresentarle in maniera originale e, a parer mio, molto efficace. 
